IJMMS 31:9 (2002) 567-576

PII. S0161171202007202

http://ijmms.hindawi.com

(c) Hindawi Publishing Corp.

\title{
ON THE UNIVALENCY FOR CERTAIN SUBCLASS OF ANALYTIC FUNCTIONS INVOLVING RUSCHEWEYH DERIVATIVES
}

\author{
LIU MINGSHENG
}

Received 20 March 2001

Let $H$ be the class of functions $f(z)$ of the form $f(z)=z+\sum_{k=2}^{+\infty} a_{k} z^{k}$, which are analytic in the unit disk $U=\{z ;|z|<1\}$. In this paper, we introduce a new subclass $B_{\lambda}(\mu, \alpha, \rho)$ of $H$ and study its inclusion relations, the condition of univalency, and covering theorem. The results obtained include the related results of some authors as their special case. We also get some new results.

2000 Mathematics Subject Classification: 30C45.

1. Introduction. Let $H$ be the class of functions of the form

$$
f(z)=z+\sum_{k=2}^{+\infty} a_{k} z^{k}
$$

which are analytic in the unit disk $U=\{z ;|z|<1\}$. Let $S$ be the subclass of $H$ consisting of univalent functions.

For the function $f(z)=\sum_{k=1}^{+\infty} a_{k} z^{k}$ and $g(z)=\sum_{k=1}^{+\infty} b_{k} z^{k}$, let $(f * g)(z)$ denote the Hadamard product or convolution of $f(z)$ and $g(z)$, defined by

$$
(f * g)(z)=\sum_{k=1}^{+\infty} a_{k} b_{k} z^{k} .
$$

Now define the function $\phi(a, c ; z)$ by

$$
\phi(a, c ; z)=\sum_{k=0}^{+\infty} \frac{(a)_{k}}{(c)_{k}} z^{k+1}, \quad(c \neq 0,-1,-2, \ldots, z \in U),
$$

where

$$
(\lambda)_{k}=\frac{\Gamma(\lambda+k)}{\Gamma(\lambda)}= \begin{cases}1, & k=0, \\ \lambda(\lambda+1) \cdots(\lambda+k-1), & k \in N=\{1,2, \ldots\} .\end{cases}
$$

It follows from [4] that

$$
z[\phi(c, c+1)]^{\prime}=c \phi(c+1, c+1)-(c-1) \phi(c, c+1) .
$$


Carlson and Shaffer [2] defined a linear operator $L(a, c)$ on $H$ by using the Hadamard product

$$
L(a, c) f=\phi(a, c ; z) * f(z), \quad f \in H .
$$

It is known in [2] that $L(a, c)$ maps $H$ into itself. If $a \neq 0,-1,-2, \ldots$, then $L(a, c)$ has a continuous inverse $L(c, a)$. Clearly, $L(a, a)$ is the unit operator. Also, if $c>a>0$, then $L(a, c)$ has the integral representation

$$
L(a, c) f(z)=\frac{\Gamma(c)}{\Gamma(a) \Gamma(c-a)} \int_{0}^{1} u^{a-2}(1-u)^{c-a-1} f(u z) d u .
$$

Ruscheweyh [7] introduced an operator $D^{\lambda}: H \rightarrow H$ defined by the Hadamard product or convolution

$$
D^{\lambda} f(z)=\frac{z}{(1-z)^{\lambda+1}} * f(z), \quad(\lambda>-1, z \in U),
$$

which implies that

$$
\begin{gathered}
D^{n} f(z)=\frac{z\left(z^{n-1} f(z)\right)^{(n)}}{n !}, \quad\left(n \in N_{0}=\{0,1,2, \ldots\}\right), \\
D^{\lambda} f(z)=L(\lambda+1,1) f(z) .
\end{gathered}
$$

Assume $\alpha>0, \mu>0, \lambda>-1, \rho<1$, a function $p(z)=1+p_{1} z+p_{2} z^{2}+\cdots$ is said to be in the class $P_{\rho}$ if and only if $p(z)$ is analytic in the unit disk $U$ and $\operatorname{Re} p(z)>\rho$, $z \in U$; a function $f(z) \in H$ is said to be in the class $B_{\lambda}(\mu, \alpha, \rho)$ if and only if it satisfies

$$
\operatorname{Re}\left[(1-\mu)\left(\frac{D^{\lambda} f(z)}{z}\right)^{\alpha}+\mu\left(D^{\lambda} f(z)\right)^{\prime}\left(\frac{D^{\lambda} f(z)}{z}\right)^{\alpha-1}\right]>\rho, \quad z \in U,
$$

where the power are understood as principle values. Below we apply this agreement. It is obvious that the subclass $B_{0}(1, \alpha, 0)$ is the subclass of Bazilevič functions, which is the subclass of univalent functions $S$, let $B(\alpha, \rho) \equiv B_{0}(1, \alpha, \rho)$. The subclass $B_{0}(1, \alpha, \rho)$ $(0 \leq \rho<1)$ has been studied by Bazilevič [1], Singh [8], respectively. $B_{0}(0, \alpha, \rho)(\rho<1)$ has been studied by Liu [5]. The subclass $B_{0}(\lambda, 1, \rho)(0 \leq \rho<1)$ has been studied by Chichra [3], Ding et al. [4], respectively.

In this paper, we study the properties of $B_{\lambda}(\mu, \alpha, \rho)$. The results obtained generalize the related works of some authors. We also obtained some new univalent criterions.

\section{Some lemmas}

LEMMA 2.1 [4]. Let $\rho<1,0<u<1, F(z) \in P_{\rho}$, then for $|z|=r<1$,

$$
\operatorname{Re}[F(z)-F(u z)] \geq \frac{2(1-\rho)(u-1) r}{(1+r)(1+u r)},
$$

and the inequality is sharp. 
LEMMA 2.2. Let $c>0, \mu>0, \rho<1, p(z)=1+p_{1} z+p_{2} z^{2}+\cdots$ be analytic in $U$. If

$$
\operatorname{Re}\left[p(z)+c \mu z p^{\prime}(z)\right]>\rho, \quad z \in U,
$$

then for $|z|=r<1$,

$$
\begin{aligned}
& \operatorname{Re}\left[p(z)+c z p^{\prime}(z)\right] \geq 2 \rho-1+\frac{2(1-\rho)}{\mu(1+r)}+2(1-\rho)\left(1-\frac{1}{\mu}\right) \frac{1}{c \mu} \int_{0}^{1} \frac{u^{1 / c \mu-1}}{1+u r} d u, \\
& \operatorname{Re}\left[p(z)+c z p^{\prime}(z)\right] \geq 2 \rho-1+\frac{1-\rho}{\mu}+2(1-\rho)\left(1-\frac{1}{\mu}\right) \frac{1}{c \mu} \int_{0}^{1} \frac{u^{1 / c \mu-1}}{1+u} d u,
\end{aligned}
$$

and these results are sharp.

Proof. Set $F(z)=p(z)+c \mu z p^{\prime}(z)$, then it follows from (2.2) that $F(z) \in P_{\rho}$, and

$$
z F(z)=(1-c \mu)[z p(z)]+c \mu z[z p(z)]^{\prime}=L\left(\frac{1}{c \mu}+1, \frac{1}{c \mu}\right)[z p(z)]
$$

that is,

$$
z p(z)=L\left(\frac{1}{c \mu}, \frac{1}{c \mu}+1\right)[z F(z)]=\frac{1}{c \mu} \int_{0}^{1} u^{1 / c \mu-1} z F(u z) d u
$$

Let $b=1 / c \mu$, then

$$
p(z)=b \int_{0}^{1} u^{b-1} F(u z) d u
$$

According to (1.5) and (2.5), we get

$$
\begin{aligned}
z[z p(z)]^{\prime} & =\left[z(\phi(b, b+1 ; z))^{\prime}\right] *[z F(z)] \\
& =b L(b+1, b+1)[z F(z)]-(b-1) L(b, b+1)[z F(z)] \\
& =b z F(z)-b(b-1) z \int_{0}^{1} u^{b-1} F(u z) d u .
\end{aligned}
$$

On the other hand, we have

$$
[z p(z)]^{\prime}=p(z)+z p^{\prime}(z)
$$

Thus

$$
\begin{aligned}
p(z)+ & c z p^{\prime}(z) \\
& =(1-c) p(z)+c[z p(z)]^{\prime} \\
& =b c F(z)+b(1-c) \int_{0}^{1} u^{b-1} F(u z) d u-b c(b-1) z \int_{0}^{1} u^{b-1} F(u z) d u \\
& =b c F(z)+b(1-b c) \int_{0}^{1} u^{b-1} F(u z) d u .
\end{aligned}
$$


If $\mu>1$, then $0<b c=1 / \mu<1$, and

$$
\begin{aligned}
\operatorname{Re}\left[p(z)+c z p^{\prime}(z)\right] & =b c \operatorname{Re}[F(z)]+b(1-b c) \int_{0}^{1} u^{b-1} \operatorname{Re}[F(u z)] d u \\
& \geq b c \cdot \frac{1-(1-2 \rho) r}{1+r}+b(1-b c) \int_{0}^{1} u^{b-1} \frac{1-(1-2 \rho) u r}{1+u r} d u \\
& =2 \rho-1+\frac{2(1-\rho)}{\mu(1+r)}+2(1-\rho)\left(1-\frac{1}{\mu}\right) \frac{1}{c \mu} \int_{0}^{1} \frac{u^{1 / c \mu-1}}{1+u r} d u
\end{aligned}
$$

If $0<\mu \leq 1$, then $b c=1 / \mu \geq 1$, so that it follows from Lemma 2.1 and (2.9) that

$$
\begin{aligned}
\operatorname{Re}\left[p(z)+c z p^{\prime}(z)\right] & =\operatorname{Re}\left[b c F(z)-b(b c-1) \int_{0}^{1} u^{b-1} F(u z) d u\right] \\
& =\operatorname{Re} F(z)+b(b c-1) \int_{0}^{1} u^{b-1} \operatorname{Re}[F(z)-F(u z)] d u \\
& \geq \frac{1-(1-2 \rho) r}{1+r}+b(b c-1) \int_{0}^{1} u^{b-1} \frac{2(1-\rho)(u-1) r}{(1+r)(1+u r)} d u \\
& =2 \rho-1+\frac{2(1-\rho)}{\mu(1+r)}+2(1-\rho)\left(1-\frac{1}{\mu}\right) \frac{1}{c \mu} \int_{0}^{1} \frac{u^{1 / c \mu-1}}{1+u r} d u .
\end{aligned}
$$

Since the function

$$
2 \rho-1+\frac{2(1-\rho)}{\mu(1+r)}+2(1-\rho)\left(1-\frac{1}{\mu}\right) \frac{1}{c \mu} \int_{0}^{1} \frac{u^{1 / c \mu-1}}{1+u r} d u
$$

is decreasing with respect to $r$, therefore

$$
\operatorname{Re}\left[p(z)+c z p^{\prime}(z)\right] \geq 2 \rho-1+\frac{1-\rho}{\mu}+2(1-\rho)\left(1-\frac{1}{\mu}\right) \frac{1}{c \mu} \int_{0}^{1} \frac{u^{1 / c \mu-1}}{1+u} d u .
$$

Note that

$$
p_{\mu, c, \rho}(z)=\frac{1}{c \mu} \int_{0}^{1} u^{1 / c \mu-1} \frac{1+(1-2 \rho) u z}{1-u z} d u,
$$

satisfies (2.2), we obtain that the inequalities (2.3) are sharp.

LEMMA 2.3 [6]. Let $p(z)=1+p_{1} z+\cdots \in P_{\rho}$, then $\left|p_{k}\right| \leq 2-2 \rho, k=1,2, \ldots$

\section{Main results}

THEOREM 3.1. Let $\alpha>0, \beta>0, \lambda \geq 0, f(z) \in H$ and

$$
\operatorname{Re}\left[\left(D^{\lambda} f(z)\right)^{\prime}\left(\frac{D^{\lambda} f(z)}{z}\right)^{\alpha-1}\right]>\frac{\lambda}{\beta+\lambda}, \quad z \in U,
$$

then $f(z)$ is univalent in $U$, that is, $B_{\lambda}(1, \alpha, \lambda /(\beta+\lambda)) \subset B(\alpha, 0)$.

Proof. Since $\lambda /(\beta+\lambda) \geq 0$, we have

$$
B\left(\alpha, \frac{\lambda}{\beta+\lambda}\right) \subset B(\alpha, 0) .
$$


Let $T=D^{\lambda} f(z)$, then $T$ is a linear and homeomorphism from $B_{\lambda}(1, \alpha, \lambda /(\beta+\lambda))$ onto $B(\alpha, \lambda /(\beta+\lambda))$, therefore it follows from (3.2) that

$$
B_{\lambda}\left(1, \alpha, \frac{\lambda}{\beta+\lambda}\right)=T^{-1} B\left(\alpha, \frac{\lambda}{\beta+\lambda}\right) \subset T^{-1} B(\alpha, 0)=B_{0}(1, \alpha, 0) \equiv B(\alpha, 0) .
$$

Hence the proof is completed.

Since $\lim _{\beta \rightarrow \infty} \lambda /(\beta+\lambda)=0$, the following corollary follows from Theorem 3.1.

COROLlary 3.2. Let $\alpha>0,0<\rho<1, \lambda \geq 0$, then

$$
B_{\lambda}(1, \alpha, \rho) \subset B(\alpha, 0) \subset S .
$$

THEOREM 3.3. Let $\mu_{2} \geq \mu_{1}>0,1>\rho_{2} \geq \rho_{1}$, then

$$
B_{\lambda}\left(\mu_{2}, \alpha, \rho_{2}\right) \subset B_{\lambda}\left(\mu_{1}, \alpha, \rho_{1}\right) .
$$

Proof. First, it is obvious that

$$
B_{\lambda}\left(\mu_{2}, \alpha, \rho_{2}\right) \subset B_{\lambda}\left(\mu_{2}, \alpha, \rho_{1}\right)
$$

Therefore we only need to verify that

$$
B_{\lambda}\left(\mu_{2}, \alpha, \rho_{1}\right) \subset B_{\lambda}\left(\mu_{1}, \alpha, \rho_{1}\right) .
$$

Let $p(z)=\left[D^{\lambda} f(z) / z\right]^{\alpha}$ for $f \in B_{\lambda}\left(\mu_{2}, \alpha, \rho_{1}\right)$, where the power are understood as principle values, then $p(z)=1+(1+\lambda) \alpha a_{2} z+\cdots$ is analytic in $U$ and

$$
\left[D^{\lambda} f(z)\right]^{\alpha}=z^{\alpha} p(z) .
$$

By taking the derivatives in the both sides of (3.8), we obtain

$$
\left(1-\mu_{2}\right)\left(\frac{D^{\lambda} f(z)}{z}\right)^{\alpha}+\mu_{2}\left(D^{\lambda} f(z)\right)^{\prime}\left(\frac{D^{\lambda} f(z)}{z}\right)^{\alpha-1}=p(z)+\frac{\mu_{2}}{\alpha} z p^{\prime}(z) .
$$

Since $f \in B_{\lambda}\left(\mu_{2}, \alpha, \rho_{1}\right)$, we have

$$
\operatorname{Re}\left[p(z)+\frac{\mu_{2}}{\alpha} z p^{\prime}(z)\right]>\rho_{1}, \quad z \in U
$$

According to Lemma 2.2, we obtain

$$
\begin{aligned}
\operatorname{Re}\left[p(z)+\frac{\mu_{1}}{\alpha} z p^{\prime}(z)\right] & \geq 2 \rho_{1}-1+\frac{2\left(1-\rho_{1}\right)}{\mu(1+r)}+2\left(1-\rho_{1}\right)\left(1-\frac{1}{\mu}\right) \frac{\alpha}{\mu} \int_{0}^{1} \frac{u^{\alpha / \mu-1}}{1+u r} d u \\
& \geq 2 \rho_{1}-1+\frac{2\left(1-\rho_{1}\right)}{\mu}+2\left(1-\rho_{1}\right)\left(1-\frac{1}{\mu}\right) \cdot \frac{1}{2}>\rho_{1}
\end{aligned}
$$


for $\mu=\mu_{2} / \mu_{1} \geq 1$. Hence it follows from (3.6) and (3.11) that $\operatorname{Re}\left[\left(1-\mu_{1}\right)\left(\frac{D^{\lambda} f(z)}{z}\right)^{\alpha}+\mu_{1}\left(D^{\lambda} f(z)\right)^{\prime}\left(\frac{D^{\lambda} f(z)}{z}\right)^{\alpha-1}\right]=\operatorname{Re}\left[p(z)+\frac{\mu_{1}}{\alpha} z p^{\prime}(z)\right]>\rho_{1}$,

that is,

$$
f \in B_{\lambda}\left(\mu_{2}, \alpha, \rho_{1}\right)
$$

Hence

$$
B_{\lambda}\left(\mu_{2}, \alpha, \rho_{1}\right) \subset B_{\lambda}\left(\mu_{1}, \alpha, \rho_{1}\right)
$$

According to Theorem 3.3 and Corollary 3.2, we have the following corollary.

Corollary 3.4. Let $\lambda \geq 0, \mu \geq 1,0<\rho<1$, then

$$
B_{\lambda}(\mu, \alpha, \rho) \subset B_{\lambda}(1, \alpha, \rho) \subset S .
$$

THEOREM 3.5. Let $\alpha>0, \lambda \geq 0, \mu>0, \rho<1$. If $f \in B_{\lambda}(\mu, \alpha, \rho)$, then for $|z|=r<1$,

$$
\begin{aligned}
& \operatorname{Re}\left[\left(D^{\lambda} f(z)\right)^{\prime}\left(\frac{D^{\lambda} f(z)}{z}\right)^{\alpha-1}\right] \geq 2 \rho-1+\frac{2(1-\rho)}{\mu(1+r)}+2(1-\rho)\left(1-\frac{1}{\mu}\right) \frac{\alpha}{\mu} \int_{0}^{1} \frac{u^{\alpha / \mu-1}}{1+u r} d u, \\
& \operatorname{Re}\left[\left(D^{\lambda} f(z)\right)^{\prime}\left(\frac{D^{\lambda} f(z)}{z}\right)^{\alpha-1}\right] \geq 2 \rho-1+\frac{1-\rho}{\mu}+2(1-\rho)\left(1-\frac{1}{\mu}\right) \frac{\alpha}{\mu} \int_{0}^{1} \frac{u^{\alpha / \mu-1}}{1+u} d u,
\end{aligned}
$$

and these results are sharp.

Proof. Let $p(z)=\left[D^{\lambda} f(z) / z\right]^{\alpha}$ for $f \in B_{\lambda}(\mu, \alpha, \rho)$, where the power are understood as principle values, then

$$
p(z)=1+(1+\lambda) \alpha a_{2} z+\cdots
$$

is analytic in $U$ and

$$
\left[D^{\lambda} f(z)\right]^{\alpha}=z^{\alpha} p(z)
$$

By taking the derivatives in the both sides of (3.18), we obtain

$$
(1-\mu)\left(\frac{D^{\lambda} f(z)}{z}\right)^{\alpha}+\mu\left(D^{\lambda} f(z)\right)^{\prime}\left(\frac{D^{\lambda} f(z)}{z}\right)^{\alpha-1}=p(z)+\frac{\mu}{\alpha} z p^{\prime}(z) .
$$

Since $f \in B_{\lambda}(\mu, \alpha, \rho)$, we have

$$
\operatorname{Re}\left[p(z)+\frac{\mu}{\alpha} z p^{\prime}(z)\right]>\rho, \quad z \in U .
$$


According to Lemma 2.2, we obtain

$$
\begin{aligned}
\operatorname{Re}\left[\left(D^{\lambda} f(z)\right)^{\prime}\left(\frac{D^{\lambda} f(z)}{z}\right)^{\alpha-1}\right] & =\operatorname{Re}\left[p(z)+\frac{1}{\alpha} z p^{\prime}(z)\right] \\
& \geq 2 \rho-1+\frac{2(1-\rho)}{\mu(1+r)}+2(1-\rho)\left(1-\frac{1}{\mu}\right) \frac{\alpha}{\mu} \int_{0}^{1} \frac{u^{\alpha / \mu-1}}{1+u r} d u, \\
\operatorname{Re}\left[\left(D^{\lambda} f(z)\right)^{\prime}\left(\frac{D^{\lambda} f(z)}{z}\right)^{\alpha-1}\right] & =\operatorname{Re}\left[p(z)+\frac{1}{\alpha} z p^{\prime}(z)\right] \\
& \geq 2 \rho-1+\frac{1-\rho}{\mu}+2(1-\rho)\left(1-\frac{1}{\mu}\right) \frac{\alpha}{\mu} \int_{0}^{1} \frac{u^{\alpha / \mu-1}}{1+u} d u .
\end{aligned}
$$

Note that,

$$
f_{\lambda, \mu, \alpha, \rho}(z)=L(1, \lambda+1)\left\{z\left[\frac{\alpha}{\mu} \int_{0}^{1} u^{\alpha / \mu-1} \frac{1+(1-2 \rho) u z}{1-u z} d u\right]^{1 / \alpha}\right\} \in B_{\lambda}(\mu, \alpha, \rho),
$$

we obtain that inequalities (3.16) are sharp.

REMARK 3.6. Setting $\lambda=0, \alpha=1$ in Theorem 3.5, we get [4, Theorem 1(ii)].

THEOREM 3.7. Let $\alpha>0, \mu>0, \lambda \geq 0, \rho_{0} \leq \rho<1$, then $B_{\lambda}(\mu, \alpha, \rho) \subset B_{\lambda}\left(1, \alpha, \rho_{1}\right) \subset S$, where

$$
\rho_{0}=1-\frac{1}{2-1 / \mu-2(1-1 / \mu)(\alpha / \mu) \int_{0}^{1}\left(u^{\alpha / \mu-1} /(1+u)\right) d u},
$$

and the constant $\rho_{0}$ cannot be replaced by any smaller one.

PROoF. Let $f(z) \in B_{\lambda}(\mu, \alpha, \rho)$, then it follows from Theorem 3.5 that

$$
\operatorname{Re}\left[\left(D^{\lambda} f(z)\right)^{\prime}\left(\frac{D^{\lambda} f(z)}{z}\right)^{\alpha-1}\right]>\rho_{1}, \quad z \in U,
$$

where

$$
\begin{aligned}
\rho_{1} & =2 \rho-1+\frac{1-\rho}{\mu}+2(1-\rho)\left(1-\frac{1}{\mu}\right) \frac{\alpha}{\mu} \int_{0}^{1} \frac{u^{\alpha / \mu-1}}{1+u} d u \\
& =1-(1-\rho)\left[2-\frac{1}{\mu}-2\left(1-\frac{1}{\mu}\right) \frac{\alpha}{\mu} \int_{0}^{1} \frac{u^{\alpha / \mu-1}}{1+u} d u\right] .
\end{aligned}
$$

Since

$$
\frac{1}{2}<\frac{\alpha}{\mu} \int_{0}^{1} \frac{u^{\alpha / \mu-1}}{1+u} d u=\frac{1}{2}+\int_{0}^{1} \frac{u^{\alpha / \mu}}{(1+u)^{2}} d u<1
$$

so that

$$
\max \left\{1, \frac{1}{\mu}\right\}>2-\frac{1}{\mu}-2\left(1-\frac{1}{\mu}\right) \frac{\alpha}{\mu} \int_{0}^{1} \frac{u^{\alpha / \mu-1}}{1+u} d u>\min \left\{1, \frac{1}{\mu}\right\}>0 .
$$


Therefore from $\rho_{0} \leq \rho<1$, we have

$$
\rho_{1}>1-\left(1-\rho_{0}\right)\left[2-\frac{1}{\mu}-2\left(1-\frac{1}{\mu}\right) \frac{\alpha}{\mu} \int_{0}^{1} \frac{u^{\alpha / \mu-1}}{1+u} d u\right]=0 .
$$

Hence it follows from (1.10) and Corollary 3.2 that $f(z) \in B_{\lambda}\left(1, \alpha, \rho_{1}\right) \subset S$ and $f(z)$ is univalent in $U$, hence

$$
B_{\lambda}(\mu, \alpha, \rho) \subset B_{\lambda}\left(1, \alpha, \rho_{1}\right) \subset S
$$

and the constant $\rho_{0}$ cannot be replaced by any smaller one from Theorem 3.5.

REMARK 3.8. Setting $\lambda=0, \alpha=1$ in Theorem 3.7, we get [4, Theorem 2]; setting $\lambda=0, \mu=1$ in Theorem 3.7, we get the result of [1].

Setting $\lambda=0, \mu=\alpha>0$ in Theorem 3.7, we have the following corollary.

COROLlary 3.9. If $f(z) \in H$, and

$$
\operatorname{Re}\left[(1-\alpha)\left(\frac{f(z)}{z}\right)^{\alpha}+\alpha f^{\prime}(z)\left(\frac{f(z)}{z}\right)^{\alpha-1}\right]>\rho_{\alpha}=\frac{(\alpha-1)(1-2 \ln 2)}{\alpha+(\alpha-1)(1-2 \ln 2)}, \quad z \in U,
$$

then $f(z)$ is univalent in $U$, and the result is sharp.

REMARK 3.10. We note that $\rho_{\alpha}<0$ for $\alpha>1$.

Setting $\mu=\alpha=3, \lambda=1$ in Theorem 3.7, we have the following corollary.

COROLlary 3.11. If $f(z) \in H$, and

$$
\operatorname{Re}\left\{z\left[\left(f^{\prime}(z)\right)^{3}\right]^{\prime}+\left(f^{\prime}(z)\right)^{3}\right\}>\frac{2-4 \ln 2}{5-4 \ln 2} \approx-0.34, \quad z \in U,
$$

then $f(z)$ is univalent in $U$.

THEOREM 3.12. Let $f(z)=z+\sum_{k=2}^{+\infty} a_{k} z^{k} \in B_{\lambda}(\mu, \alpha, \rho)$, then

$$
\left|a_{2}\right| \leq \frac{2-2 \rho}{(1+\lambda)(\alpha+\mu)}
$$

and the inequality is sharp, with the extremal function defined $f_{\lambda, \mu, \alpha, \rho}(z)$ by (3.22).

Proof. Since $f(z)=z+\sum_{k=2}^{+\infty} a_{k} z^{k} \in B_{\lambda}(\mu, \alpha, \rho)$, we obtain

$$
\begin{gathered}
\operatorname{Re}\left[(1-\mu)\left(\frac{D^{\lambda} f(z)}{z}\right)^{\alpha}+\mu\left(D^{\lambda} f(z)\right)^{\prime}\left(\frac{D^{\lambda} f(z)}{z}\right)^{\alpha-1}\right] \\
=\operatorname{Re}[1+(1+\lambda)(\alpha+\mu) z+\cdots]>\rho .
\end{gathered}
$$

Therefore, it follows from Lemma 2.3 that

$$
\left|(1+\lambda)(\alpha+\mu) a_{2}\right| \leq 2-2 \rho
$$


or

$$
\left|a_{2}\right| \leq \frac{2-2 \rho}{(1+\lambda)(\alpha+\mu)}
$$

Note that $f_{\lambda, \mu, \alpha, \rho}(z)=z+((2-2 \rho) /(1+\lambda)(\alpha+\mu)) z^{2}+\cdots \in B_{\lambda}(\mu, \alpha, \rho)$, we obtain that inequality (3.32) is sharp.

REMARK 3.13. Setting $\lambda=0, \mu=1$ in Theorem 3.12, we get [8, Theorem 6].

TheOrem 3.14 (covering theorem). Let $\alpha>0, \mu>0, \lambda \geq 0, \rho_{0} \leq \rho<1, f(z) \in$ $B_{\lambda}(\mu, \alpha, \rho)$, then the unit disk $U$ is mapped on a domain that contain the disk $|w|<r_{1}$, where $\rho_{0}$ defined by (3.23) and

$$
r_{1}=\frac{(1+\lambda)(\alpha+\mu)}{2-2 \rho+2(1+\lambda)(\alpha+\mu)} .
$$

Proof. Let $w_{0}$ be any complex number such that $f(z) \neq w_{0}$ for $z \in U$, then $w_{0} \neq 0$ and

$$
\frac{w_{0} f(z)}{w_{0}-f(z)}=z+\left(a_{2}+\frac{1}{w_{0}}\right) z^{2}+\cdots
$$

is univalent in $U$ by Theorem 3.7, so

$$
\left|a_{2}+\frac{1}{w_{0}}\right| \leq 2
$$

Therefore according to Theorem 3.12, we obtain

$$
\left|w_{0}\right| \geq \frac{(1+\lambda)(\alpha+\mu)}{2-2 \rho+2(1+\lambda)(\alpha+\mu)}=r_{1} .
$$

Hence we have completed the proof.

Setting $\lambda=0$ and $\mu=1$ in Theorem 3.14, we have the following corollary.

COROLlary 3.15 (covering theorem). Let $f(z) \in B_{0}(1, \alpha, \rho)$ with $\mu>0,0 \leq \rho<1$, then the unit disk $U$ is mapped on a domain that contain the disk $|w|<(1+\alpha) /(4-$ $2 \rho+2 \alpha)$.

ACKNOWLEDGMENT. This research was supported by the National Natural Science Foundation of China grant no. 19971029.

\section{REFERENCES}

[1] I. E. Bazilevič, On a case of integrability in quadratures of the Loewner-Kufarev equation, Mat. Sb. (N.S.) 37(79) (1955), 471-476.

[2] B. C. Carlson and D. B. Shaffer, Starlike and prestarlike hypergeometric functions, SIAM J. Math. Anal. 15 (1984), no. 4, 737-745.

[3] P. N. Chichra, New subclasses of the class of close-to-convex functions, Proc. Amer. Math. Soc. 62 (1976), no. 1, 37-43.

[4] S. S. Ding, Y. Ling, and G. J. Bao, Some properties of a class of analytic functions, J. Math. Anal. Appl. 195 (1995), no. 1, 71-81. 
[5] M. S. Liu, The radius of univalence for certain class of analytic functions, Boundary Value Problems, Integral Equations and Related Problems (Beijing/Chengde, 1999) (L. Jianke and W. Guochun, eds.), World Scientific Publishing, New Jersey, 2000, pp. 122-128.

[6] C. Pommerenke, Univalent Functions, Vandenhoeck \& Ruprecht, Göttingen, 1975.

[7] S. Ruscheweyh, New criteria for univalent functions, Proc. Amer. Math. Soc. 49 (1975), 109-115.

[8] R. Singh, On Bazilevič functions, Proc. Amer. Math. Soc. 38 (1973), 261-271.

Liu Mingsheng: Department of Mathematics, South China normal University, GuANGZHOU 510631, CHINA

E-mail address: 1iumsh@scnu.edu.cn 


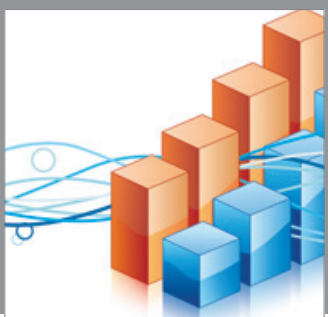

Advances in

Operations Research

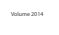

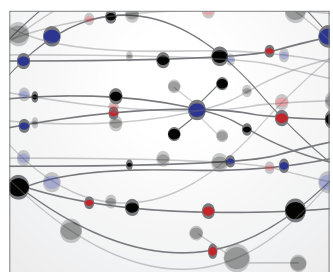

\section{The Scientific} World Journal
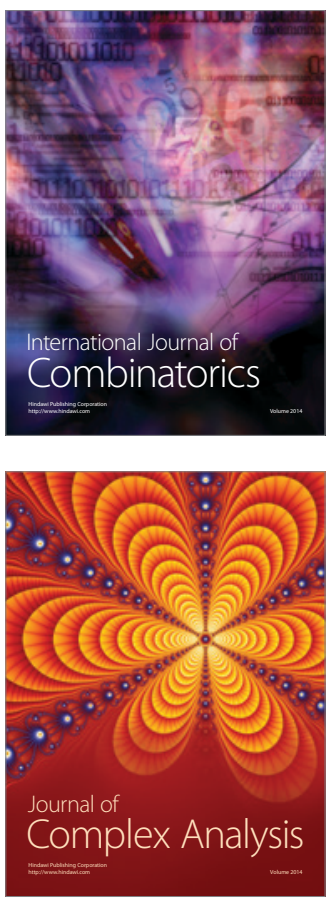

International Journal of

Mathematics and

Mathematical

Sciences
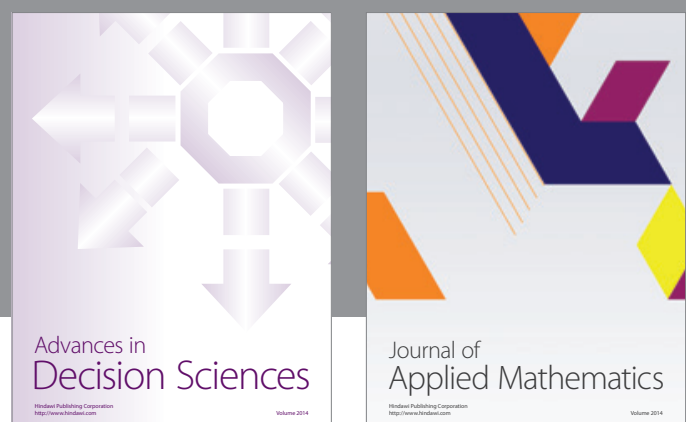

Journal of

Applied Mathematics
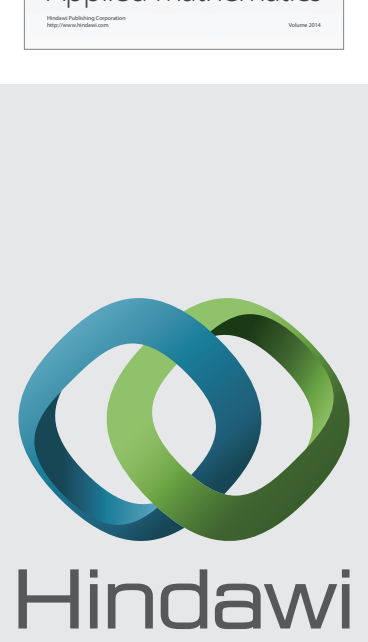

Submit your manuscripts at http://www.hindawi.com
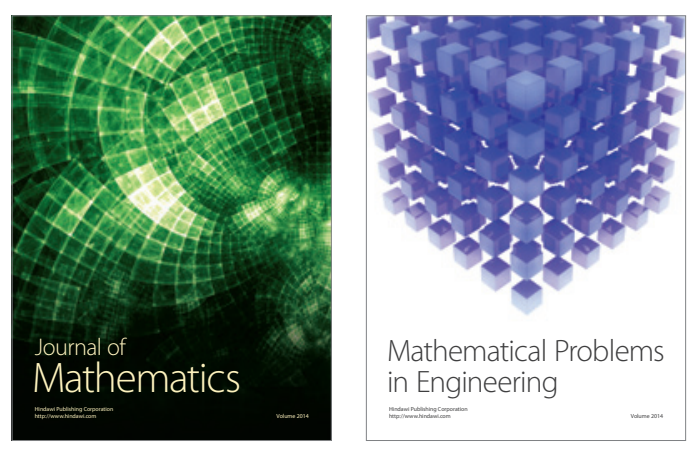

Mathematical Problems in Engineering
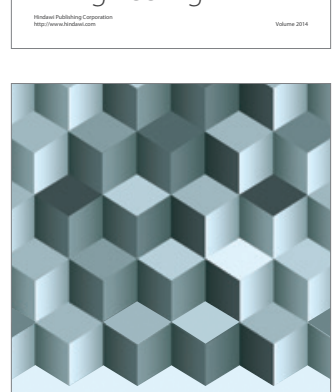

Journal of

Function Spaces
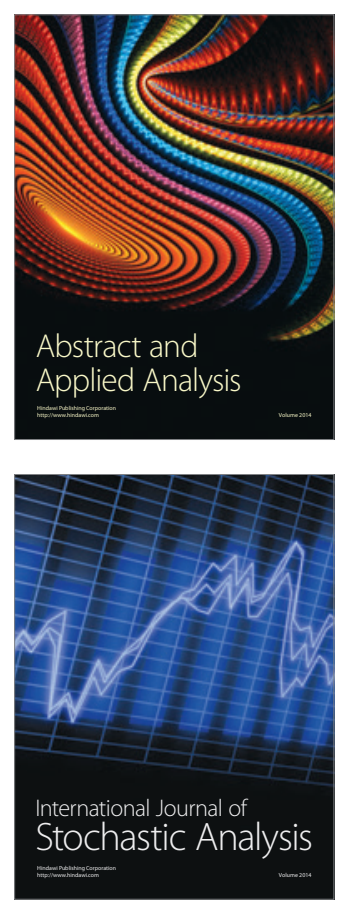

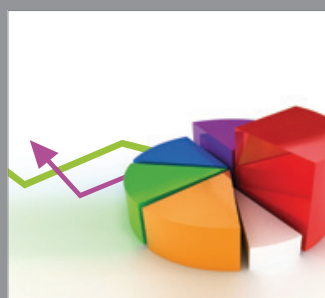

ournal of

Probability and Statistics

Promensencen
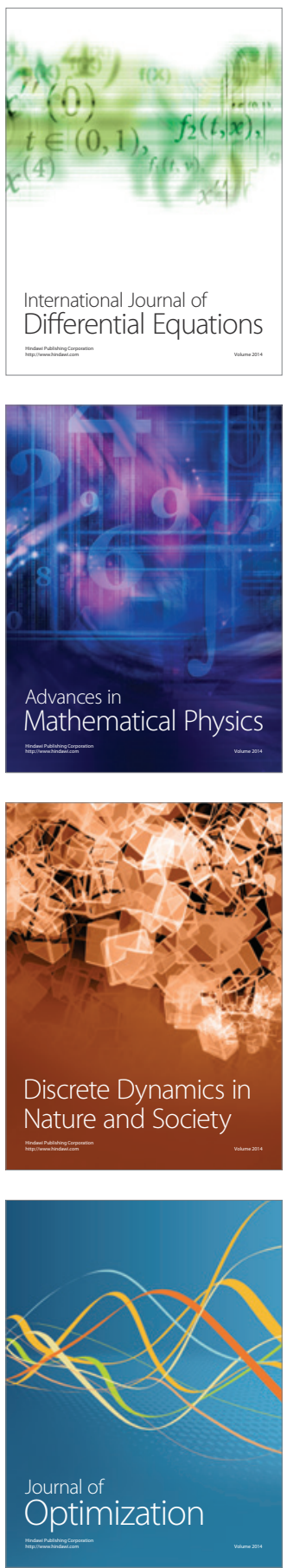